\title{
Possibilities of using thermoelectric generators (TEG) for producing electrical energy by living subjects
}

\author{
Štěpán Remeš, Jan Havlík \\ Faculty of Electrical Engineering \\ Czech Technical University in Prague \\ Prague, Czech Republic \\ xhavlikj@fel.cvut.cz
}

\begin{abstract}
This work aims to study of possibilities of a realization a device, which will be able to harvest electric energy from human body heat. The concept was proved in different conditions - without physical activity, during walking and running. Based on statistical analysis, the possibility of energy harvesting using a thermoelectric generator is discussed. The amount of harvested energy is evaluated based on experiments.
\end{abstract}

Keywords - energy harvesting; thermoelectric generators; Peltier cells; human body waste; Seebeck effect

\section{INTRODUCTION}

In recent years, many companies focused on producing electrical energy from sunlight, wind etc. [1]. But there is also one significant possible source of energy which could be transformed into electrical energy and is still not explored as sources mentioned above - living subjects.

There are already many tries on how to harvest electric energy from living subjects. Some of them need some special conditions like device, which create electrical energy from every step by the piezoelectric cell [2], or device which generates electricity from every vibration of a body during walking or moving by oscillating of permanent magnets near a coil [3]. There are also devices which don't need any special conditions like a device from Swiss engineers who try to create electric energy from blood flow by microturbine inside of veins or arteries [4]. The study from Athens university presents energy harvesting by electromagnetic induction from the pulsation of the artery [5]. Unfortunately, both last principles are invasive. The other possibility is using thermoelectric generators (TEG), which can harvest electric energy from temperature differences. The harvesting of electric energy from human body using TEGs is a modern approach which seems suitable for powering of small wearable or portable devices $[6,7,8]$.

The aim of this research is a study of practical possibilities of energy harvesting using TEGs and to evaluate the amount of generated energy during a physical activity and relaxation.

\section{THERMOELECTRIC GENERATOR}

A thermoelectric generator (TEG) is a device which can harvest electric energy from temperature differences by one or (usually) more Peltier cells.

\section{A. Peltier cells}

Peltier cells generally consist of PN semiconductors with specific collocation working on the principle of Peltier-Seebeck effect. These two semiconductors are not connected in traditional diode collocation. Still, they are joined by a copper bridge in serial connection with another Peltier cell as it is shown on Fig. 1.

The using of Peltier cells should not be only to generate electric energy. If there are connected a source of electrical energy to the Peltier cell, it works just the opposite way - one side is heating up, and the other side is cooling down. This is the effect which could be used in a fridge, for example. Unfortunately, the Peltier cells have low efficiency. That's why it has other uses - it is possible to realize light and small structures which can cool microcontrollers or chips, for example. [6]

\section{B. Wearable Thermoelectric Generator}

Unlike traditional TEGs which are consist of an array of Peltier cells between 2 ceramic plates, wearable TEG is not covered by anything, just embedded in flexible mass. It is possible to see raw small copper plates (bridges). Thus, it is possible to bend it and to achieve the desired shape for best adhesion to a body surface.

\section{TESTING OF THERMOELECTRIC GENERATOR}

For testing of TEG, we have flexible Peltier cell field consisted of approximately of 180 cells connected in series. Each of them is about $8 \mathrm{~mm}$ long, $2 \mathrm{~mm}$ wide and $3 \mathrm{~mm}$ high. Whole TEG dimensions are $85 \times 65 \times 3 \mathrm{~mm}^{3}$.

\section{A. Preparation}

Before the evaluation of the TEG usability, we had to found a load for which the harvested power is maximal. The dependency of the output power on the load has been evaluated. The highest output power 
was with resistance load of $4,7 \Omega$ as is shown in Fig. 2. Thus, all experiments were done with this load.

Figure 1. Two Peltier cells connected by a copper bridge [1]



Figure 2. The dependency of generated power on the load

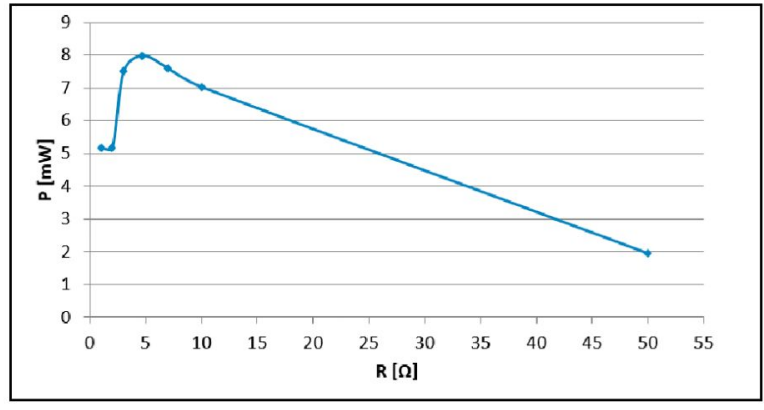

A portable voltage and activity logger with specified load was realized for evaluating of the TEG. It was realized based on Arduino Nano development board; the activity was sensed using MPU92/65 module containing accelerometer, gyroscope, magnetometer and thermometer. The data are stored to the SD card (SD card module connected to Arduino). The sampling frequency of the device is $70 \mathrm{~Hz}$ to get relevant data. With this setup, it was possible to evaluate a power generated by TEG depending on physical activity.

\section{B. Measurement on real subjects}

Each measure takes about 30 minutes and consists of run session or run-stop-run session, walking session etc. On the charts below, the typical behaviour of signals acquired during walking (Fig. 3) and running (Fig. 6) are shown. The experiment consists of activity with breaks. A blue curve is the sum of absolute values from 3-axes gyroscope in [g], and an orange one is the output power in [mW].

In all cases, the generated voltage was between $0 \mathrm{mV}$ and $200 \mathrm{mV}$. But as it is shown, the generated energy during running is about twice higher than during breaks (typically during resting without physical activity). Based on realized observations, it is difficult to say what is a resting output voltage because it depends on ambient conditions. Inside a building (with room temperature) it generates about $20 \mathrm{mV}$ when the subject is chilled. And with the same subject and same ambient temperature outside, but with the fresh wind (high airflow) it is possible to generate up to $100 \mathrm{mV}$.

\section{RESULTS AND DISCUSSION}

In the last chart, it is shown that main impact to harvested energy has the cold air around the TEG; and how well is the outer side of TEG cooled down, because after stopping the physical activity (walking or running) the generated power was twice lower almost immediately. The impact of inner side heating by intensive physical activity - running is also distinguishable from the charts; there is possible to see an increase of generated power almost after 5 minutes of running.

Figure 3. Measurement of walking with breaks with showing acceleration [g] (blue line) and generated power [ $\mathrm{mW}]$

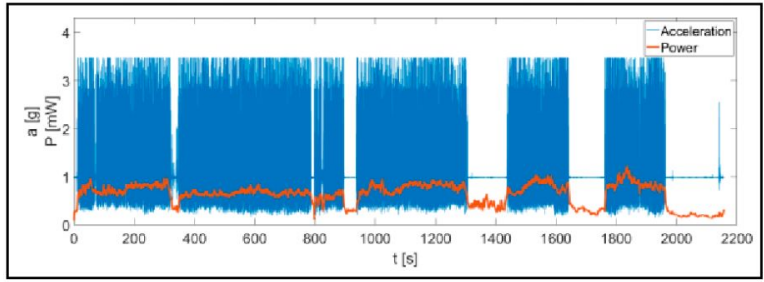

Figure 4. Generated power by the subject during walking-resting periods (above) and for physical activity (below)

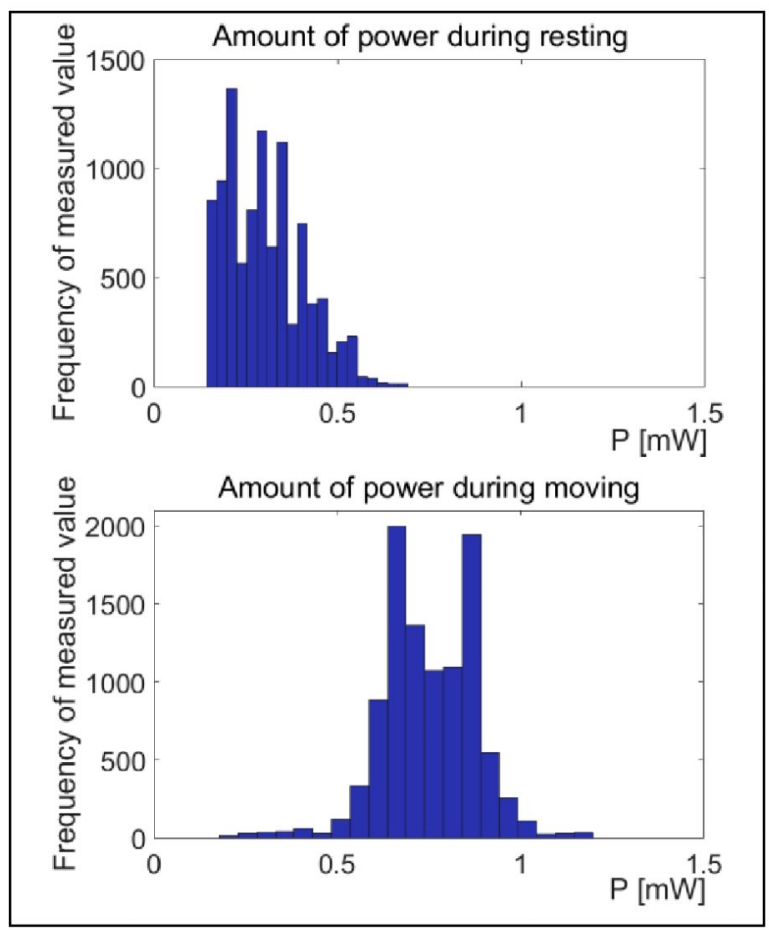

Figure 5. Box plots of the medians of chosen random samples of generated power during walking

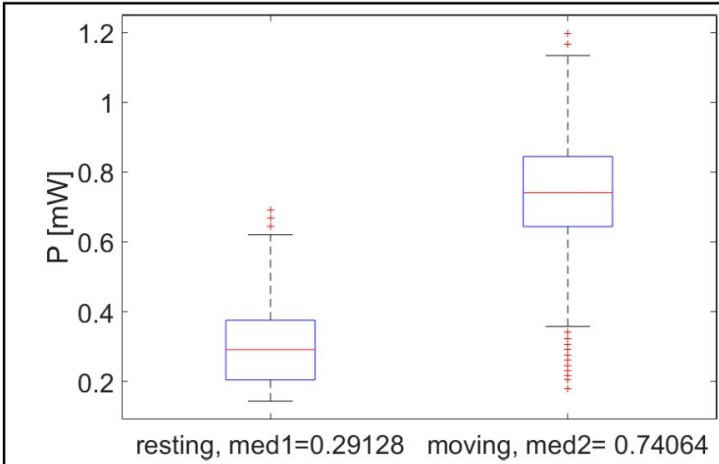

After all observations, it could be judged that the human body is a very consistent source of power. The TEG efficiency strongly depends on the proper cooling of the outer side of TEG. It could also be 
stated that it is possible to increase the amount of harvested energy by intensive physical activity by muscle heating. Still, this effect is visible after a longer time than just moving with the TEG and cooling the outer side.

Figure 6. Measurement of running with breaks with showing acceleration [g] (blue line) and generated power [ $\mathrm{mW}]$

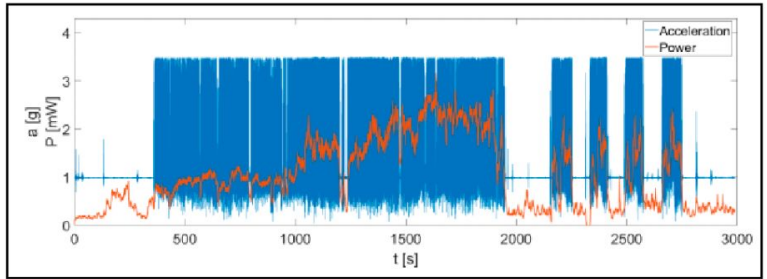

Figure 7. Generated power by the subject during running-resting periods (above) and for physical activity (below)

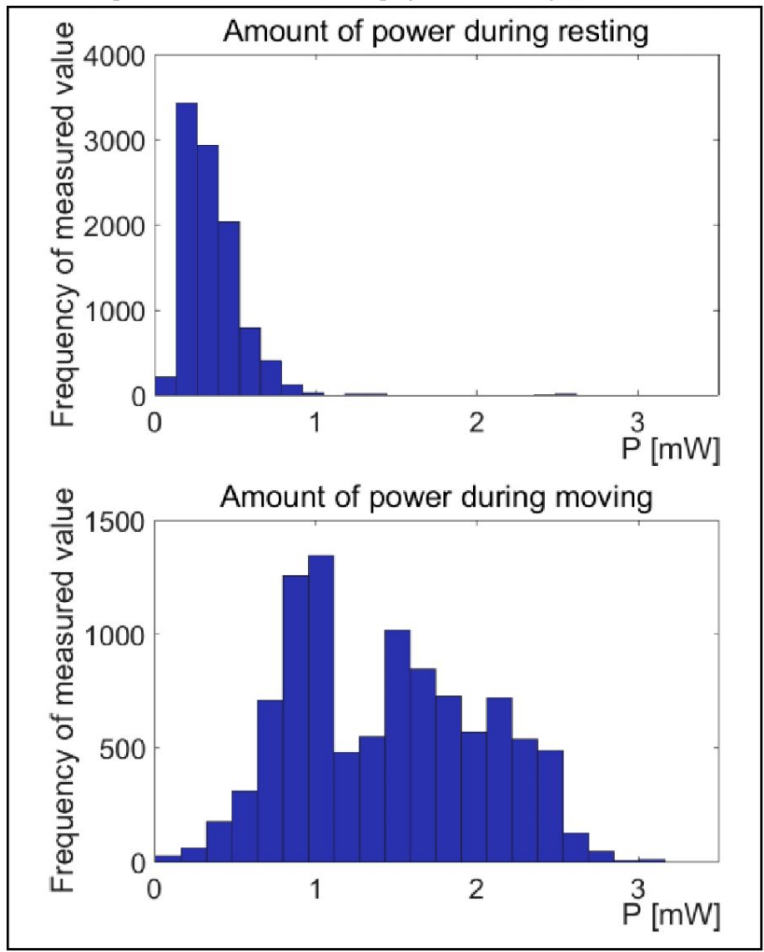

Figure 8. Box plots of the medians of chosen random samples of generated power during running

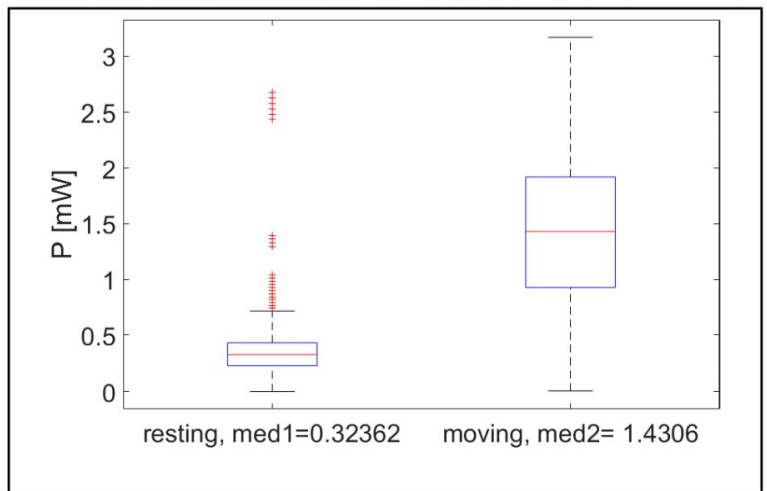

Based on this data, a hypothesis that an amount of harvested energy depends on physical activity has been proved using Wilcoxon's test (the test approves if mean values of the data are different. A dataset consisting of 10000 random samples of values marked as "generated during resting" and 10000 random samples of values marked as "generated during moving" has been collected (see Fig 4 for walking and Fig 7 for running).

The hypothesis that the mean values for physical activity and resting are the same was strongly rejected by Wilcoxon's test $(p<0.001)$. Box plots of the medians of chosen random samples which describe the difference between moving and resting subjects are shown in Fig. 5 for walking and Fig. 8 for running.

\section{CONCLUSION}

The TEGs are usable for energy harvesting from living subjects. The amount of the energy which is possible to harvest from human body during physical activity is typically in miliwats. The output power of the TEG is strongly dependent on the cooling of the outer side of the TEG resp. on air flow on the outer side. The amount of generated energy is significantly higher during physical activity (walking or running) than during relaxation.

After all experiments, it could be said that amount of harvested energy is not as high as from example solar panel, but it is very stable. And, and it is substantial, this energy is generated every day of the whole life.

\section{ACKNOWLEDGEMENT}

This work has been supported by the grant no. SGS20/167/OHK3/3T/13 of the Czech Technical University in Prague.

\section{REFERENCES}

[1] C. Aawell: These 5 IoT Energy-Harvesting Options Stand Out "in the Field", ElectronicDesign, 2018, [online, cited March 14, 2020].

Available from: https://www.electronicdesign.com/powermanagement/article/21806752/these-5-iot-energyharvestingoptions-stand-out-in-the-field

[2] J. Zhao, Z. You: A Shoe-Embedded Piezoelectric Energy Harvester for Wearable Sensors. Sensors, vol. 14, pp. 12497 12510, Basel, Switzerland, 2014.

[3] K. Ylli, D. Hoffmann, A. Willmann, P. Becker, B. Folkmer, Y. Manoli: Energy harvesting from human motion: exploiting swing and shock excitations. Smart Materials and Structures, vol. 24, num. 2, 2015.

[4] D. Savenije, 5 ways you can use the human body to generate electricity. UtilityDive, 2014. [online, cited March 14, 2020], Available from: https://www.utilitydive.com/news/5-waysyou-can-use-the-human-body-to-generate-electricity/280709/

[5] M. Angelika-Nikita, N. Hadjigeorgiou, CH. Manopoulos, J Georgiou: Converting energy captured from blood flow into usable electric power: design optimisation. Medical Physics, Cornell University, 2018. [online, cited March 14, 2020]. Available from: https://arxiv.org/abs/1809.10929

[6] A. R. M. Siddique, S. Mahmud, B. van Heyst: A Review of the State of the Science on Wearable Thermoelectric Power Generators (TEGs) and their Existing Challenges. Renewable and Sustainable Energy Reviews, vol. 73. pp. 730-744, 2017. ISSN 1364-0321.

[7] M. Zhou, et al. A Review on Heat and Mechanical Energy Harvesting from Human - Principles, Prototypes and Perspectives. Renewable and Sustainable Energy Reviews, vol. 82, pp. 3582-3609, 2018. ISSN 1364-0321.

[8] M. Thielen et al. Thermal Harvesting Potential of the Human Body. Journal of Electronic Materials, vol. 47, no. 6., pp. 3307-3313, 2018. ISSN 0361-5235.

[9] J. Reichl, M. Všetička: Peltierův jev [in Czech], 2020, [online, cited March 14, 2020] Available from: http://fyzika.jreichl.com/main.article/view/911-peltieruv-jev 